\title{
Stimuli-Responsive PNIPAM Based Copolymers: Modeling and Light Scattering Investigations
}

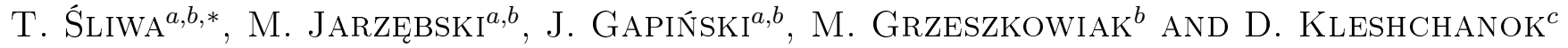 \\ ${ }^{a}$ Adam Mickiewicz University, Faculty of Physics, Umultowska 85, 61-614 Poznań, Poland \\ ${ }^{b}$ NanoBioMedical Centre, Adam Mickiewicz University, Umultowska 85, 61-614 Poznań, Poland \\ ${ }^{c}$ SABIC Europe, Science and Technology Centre, Koolwaterstofstraat 1, 6161 Geleen, The Netherlands
}

\section{(Received October 26, 2013)}

Temperature dependent behavior of poly $(N$-isopropylacrylamide) (PNIPAM) copolymers was investigated in three buffer-stabilized environments. The tests were performed in thermostabilized goniometer by dynamic and static light scattering. From the dynamic light scattering hydrodynamic radius was obtained. Based on the static light scattering data, calculations of the particle radius from the form factor model were done. Depending of the kind of copolymer, the different material response to temperature was observed. Also the characterization of dry particles was done by scanning electron microscope. These scanning electron microscopy micrograms showed the possibility of particles to aggregation.

DOI: 10.12693 /APhysPolA.125.1236

PACS: $82.35 . J k$

\section{Introduction}

Multi-stimuli responsive polymer nanoparticles are now widely studied as a promising material in medical applications. The unique properties of poly $(N$-isopropylacrylamide) (PNIPAM)-based polymers and copolymers indicate them as an innovative drug carrier for drug delivery systems [1], diagnostic substance carriers, and also as biosensors (i.e. nanothermometer) [2].

PNIPAM-based polymers exhibit changes in conformation as a response to external stimuli such as changes of $\mathrm{pH}$, temperature, ionic strength, salt concentration, electric and magnetic field, light or ultrasounds (Meid et al. [3]). Materials which react specifically to such changes in their environment are also called "smart materials".

The water solution of poly( $N$-isopropylacrylamide)-based polymers and copolymers are also categorized as a hydrogel. Hydrogel can be defined as a highly swollen three-dimensional hydrophilic polymer network. The properties of such systems differ from the properties of both raw materials: solid and liquid [3]. Hydrophilic polymer chains crosslinked in constrained volumes form special kind of particles called microgels. Typically, microgels in swollen state have a spherical shape with diameter from $50 \mathrm{~nm}$ up to $10 \mu \mathrm{m}$. Pich and Richtering [4] propose to classify aqueous solution of microgels as porous particles. Often, multi-stimuli responsive polymer whose average diameters are less than a $\mu \mathrm{m}$ are also called nanogels [5].

PNIPAM is a thermo- and pH-responsive polymer with a volume phase transition temperature, called lower crit-

*corresponding author; e-mail: Tomasz.Sliwa@amu.edu.pl ical solution temperature (LCST), around $32{ }^{\circ} \mathrm{C}$ which is near to human physiological temperature. PNIPAM is a polymeric chain with a hydrophobic backbone containing hydrophilic side-groups like $-\mathrm{CONH}_{2}$. Below the critical temperature, PNIPAM particles are in swollen state.

The hydrophilic interaction between the solution and polymer chains dominate over the interaction between the PNIPAM chains, thus the particles are well soluble in water. Above the critical temperature the interaction between polymer chains becomes stronger. The attractive forces between chains induce the particle contraction. Because particles become hydrophobic at temperatures higher than LCST, water that was bound inside the particles is expelled to the surroundings. This phenomenon is based on the Donnan potential which is connected to the degree of the dissociation caused by the $\mathrm{pH}$ of the surrounding. The shrinkage of the whole particle of pure PNIPAM undergoes very slowly and needs over a month to reach the equilibrium state. Functionalization of PNIPAM by chemical groups during the synthesis allows to modify PNIPAM copolymer response to the stimuli.

In the early studies of the PNIPAM polymers the thermally-induced sorption and desorption of human gamma globulin with PNIPAM particles was reported [6]. Furthermore, Yasui et al. showed application of PNIPAM to control the trypsin enzyme activity by temperature [7]. Studies performed by Matsumaru et al. revealed negative results on PNIPAM cytotoxicity [8]. The test on mice proved no acute toxicity. Also tests in two different mammalian cell lines showed no significant genotoxic response, which confirms biocompatibility of PNIPAM polymers [9]. In summary, the PNIPAM based copolymers are attractive as an innovative solution for targeted therapy (i.e. drug carrier) and also as model systems in colloid science. Our interest focuses on finding physical models describing the susceptibility of PNIPAM-based polymers to changes of $\mathrm{pH}$ and temperature. To real- 
ize this study, their hydrodynamic radius was measured in different environmental conditions using photon correlation spectroscopy (PCS). Additionally, static light scattering (SLS) was applied to measure form factors of PNIPAM microgel particles and pictures of dried particles were obtained using scanning electron microscopy (SEM).

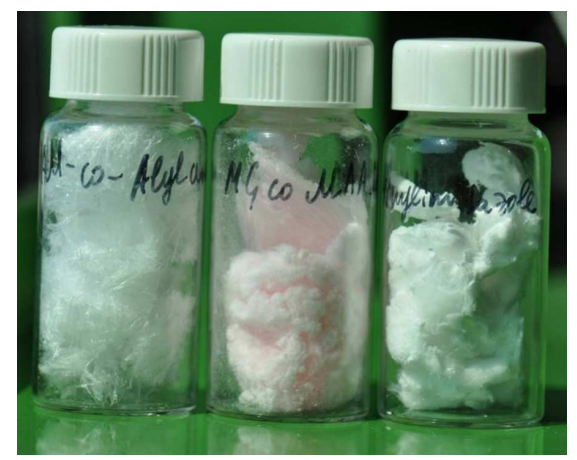

Fig. 1. Dry PNIPAM copolymers. From the left: PNIPAM-ALA, PNIPAM-MAA, PNIPAM-Vim.

\section{Sample preparation}

Synthesis of three PNIPAM copolymers was based on procedure given by Ito et al. [10] and Santos et al. [11]. Microgels were prepared from $N$-isopropylacrylamide (NIPAM) and cross-linker BIS $(N, N$ -methylenebisacrylamide). PNIPAM-co-MAA was made using methacrylic acid and was dyed by methacryloxyethyl thiocarbamyl rhodamine B. The initiator of polymerization was potassium persulfate (KPS). PNIPAM-co-Vim was made of 1-vinylimidazole and cationic surfactant dodecylethyl dimethyl ammonium bromide (DEDAB). PNIPAM-co-ALA was made of allylamine. The initiator of polymerization for PNIPAM-co-Vim and PNIPAM-co-ALA was V50 (2,2'-azobis(2-amidinopropane) dihydrochloride)). The procedure was carried out for $6 \mathrm{~h}$ under a nitrogen stream and constant stirring at $300 \mathrm{rpm}$. All PNIPAM microgel solutions were centrifuged and freeze-dried.

\section{Experimental details}

The samples of PNIPAM copolymers were initially dissolved in deionized water at the concentration of $\approx$ $1 \mathrm{mg} \mathrm{mL}^{-1}$ (batch solution). For light scattering experiments small portions of batch solutions were diluted to final concentration of $0.02 \mathrm{mg} \mathrm{mL}^{-1}$ with one of the three buffers: buffer $\mathrm{pH}=4.5$ was composed of acetic acid and sodium hydroxide solution, $\mathrm{pH}=6.0$ was made of imidazole and sodium chloride solution, $\mathrm{pH}=9.5$ was made of ammonia and sodium chloride solution.

The PCS and SLS measurements were performed in a goniometer (ALV, Germany) thermostabilized with the accuracy of 0.1 degree. The three PNIPAM copolymers were measured at the temperatures of $20^{\circ} \mathrm{C}$ and $40^{\circ} \mathrm{C}$. Laser wavelength was $\lambda=633 \mathrm{~nm}$. The scattered intensity was collected at the angle of $90^{\circ}$ for PCS and in the range $20-150^{\circ}$ for SLS by the avalanche photodiode (SPCM-AQR) of high quantum efficiency. The normalized scattered light fluctuations time autocorrelation functions $g^{(2)}(t)$ were calculated by the ALV/5000E correlator. The Siegert relation (Eq. (3.1)) was used to calculate the first order correlation function $g^{(1)}(t)$ :

$$
g^{(2)}(t)=1+\beta\left|g^{(1)}(t)\right|^{2},
$$

where $\beta$ is the coherence factor. In the simple case of noninteracting monodisperse particles $g^{(1)}(t)$ is a monoexponential function (3.2):

$$
g^{(1)}(t)=\exp (-\Gamma t),
$$

where $\Gamma$ is related to the collective diffusion coefficient (D) by Eq. (3):

$$
\Gamma=D q^{2},
$$

with $q$ being the magnitude of the scattering vector

$$
q=\frac{4 \pi n_{0}}{\lambda} \sin \frac{\theta}{2}
$$

and $n_{0}-$ solvent refractive index, $\theta-$ scattering angle.

The hydrodynamic radius $\left(R_{\mathrm{h}}\right)$ could be determined from the Stokes-Einstein Eq. (3.5):

$$
D=\frac{k_{\mathrm{B}} T}{6 \pi \eta R_{\mathrm{h}}}
$$

where $k_{\mathrm{B}}$ is the Boltzmann constant and $\eta$ - the solvent viscosity (temperature dependent).

Since the samples turned out to be nicely monodisperse (conclusion from the CONTIN analysis), $R_{\mathrm{h}}$ values were obtained from cumulant analysis.

The scattered light intensity profiles $I(q)$ obtained from SLS were treated as form factors (after normalization) because at such high dilution no interactions were expected that would lead to a distinct structure factor. The experimental data were fitted with the form factor model of a double-coated sphere. Such a model was supposed to partially account for spherically symmetric gradient of the refractive index in the microgel particle.

To check the actual size of the collapsed state of the microgel particles SEM pictures were made using the JEOL, JSM 7001F TTLS instrument. The etched surfaces were coated with gold/palladium (80:20) using sputter coater/turbo evaporator (Quorum Technologies Q150T ES) by $60 \mathrm{~s}$ to provide an electrically conductive thin film reducing the thermal damage and charging of the samples. For SEM imaging of micrographs the accelerating voltage $15 \mathrm{kV}$ and SEI secondary electron mode was used. The samples, originating from $1 \mathrm{mg} \mathrm{mL}^{-1}$ concentration solution, were dried at the temperature over $50^{\circ} \mathrm{C}$ to dry basis. Next, they were covered with platinum layers $(10 \mathrm{~nm})$ and placed inside the instrument.

\section{Results and discussion}

The results of PCS measurements have been presented in Figs. $2\left(20^{\circ} \mathrm{C}\right)$ and $3\left(40^{\circ} \mathrm{C}\right)$. Additionally, relative 
changes of $R_{\mathrm{h}}$ have been shown in Fig. 4 . With the exception of one case, the PNIPAM copolymer microgels at all three $\mathrm{pH}$ conditions shrank substantially upon heating from 20 to $40^{\circ} \mathrm{C}$. The most regular decrease was observed for PNIPAM-ALA, where the final hydrodynamic radius at $40{ }^{\circ} \mathrm{C}$ was almost the same for all three buffered solutions. PNIPAM-MAA results showed the biggest change in hydrodynamic radius for $\mathrm{pH}=4.5$ solution and the smallest for $\mathrm{pH}=9.5$. The biggest observed change in hydrodynamic radius for PNIPAM-Vim was for $\mathrm{pH}=6$. The $\mathrm{pH}=9.5$ conditions caused a slight increase of $R_{\mathrm{h}}$ for this copolymer.

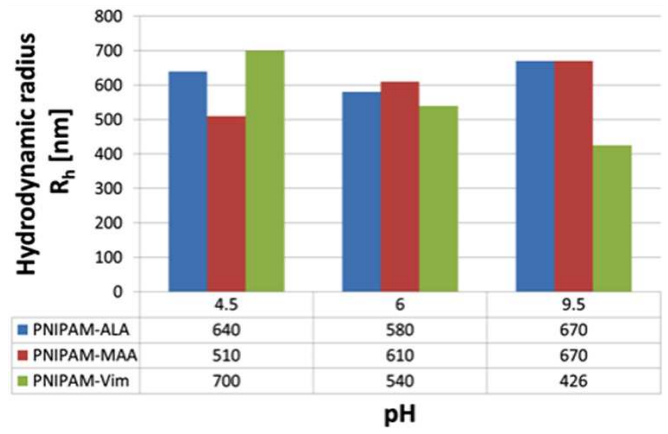

Fig. 2. Hydrodynamic radius $\left(R_{\mathrm{h}}\right)$ changes of PNIPAM-ALA, PNIPAM-MAA, PNIPAM-Vim in different $\mathrm{pH}$ at $20^{\circ} \mathrm{C}$.

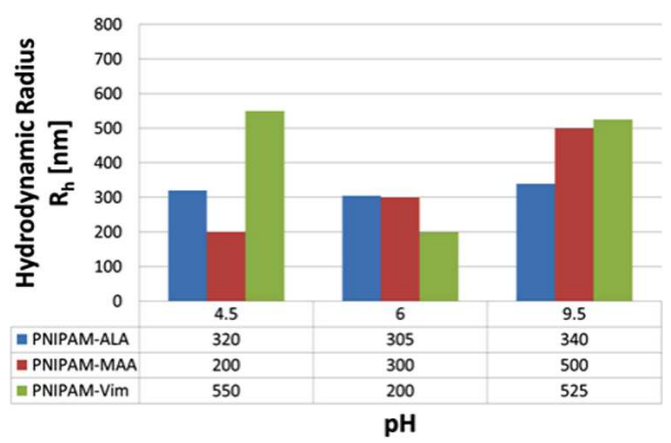

Fig. 3. Hydrodynamic radius $\left(R_{\mathrm{h}}\right)$ changes of PNIPAM-ALA, PNIPAM-MAA, PNIPAM-Vim in different $\mathrm{pH}$ at $40{ }^{\circ} \mathrm{C}$ radius.

The SLS data fitted with the core-double shell model gave the additional information about the behavior of PNIPAM copolymers caused by the $\mathrm{pH}$ and temperature (see Table). Shrinkage of PNIPAM-ALA pH $=6$ and 9.5 and PNIPAM-MAA $\mathrm{pH}=4.5$ and 6 particles is caused mainly by collapse of the shell regions. After heating the shells were collapsed. The heating of PNIPAM-Vim causes the shrinkage of core for all three $\mathrm{pH}$ environments, however the second shell structure appears. Shells have an influence on the results obtained by DLS, because of free polymer chains which create the star-like structure in external regions of particles. Free chains mostly slow-down the Brownian movement of particles which affects on the calculation of particle hydrodynamic radius.

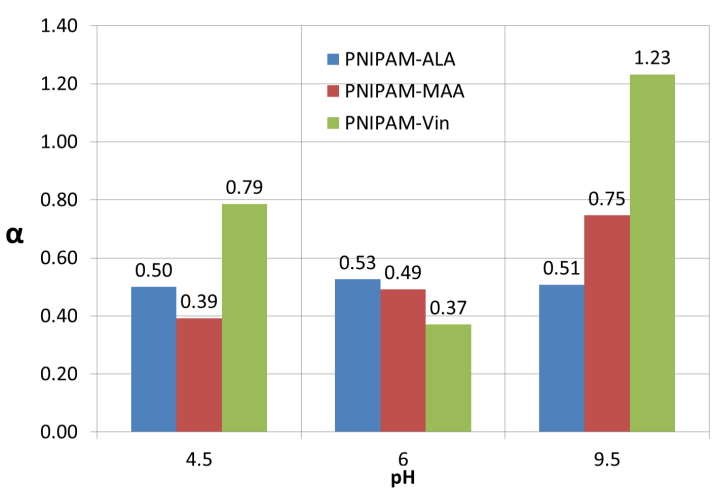

Fig. 4. The $\alpha$ parameter of PNIPAM-ALA, PNIPAM-MAA, PNIPAM-Vim changes in different $\mathrm{pH}$.

Results obtained from form factor model

TABLE (double-coated sphere).

\begin{tabular}{c|c|c|c|c|c|c}
\hline \hline PNIPAM & Temp. & $\mathrm{pH}$ & $\begin{array}{c}R \text { (core } \\
+ \text { shell1 } \\
+ \text { shell2) }\end{array}$ & core & shell1 & shell2 \\
\hline ALA & 20 & 4.5 & 478 & 380 & 56 & 42 \\
& & 6 & 509 & 374 & 135 & $\times$ \\
& \multirow{2}{*}{40} & 9.5 & 405 & 297 & 82 & 26 \\
& & 6 & 260 & 200 & 60 & $\times$ \\
& & 9.5 & 262 & 263 & $\times$ & $\times$ \\
MAA & 20 & 4.5 & 466 & 293 & $\times$ & $\times$ \\
& & 6 & 610 & 418 & 143 & 48 \\
& & 9.5 & 569 & 409 & 82 & 78 \\
& 40 & 4.5 & 201 & 201 & $\times$ & $\times$ \\
& & 6 & 203 & 203 & $\times$ & $\times$ \\
& & 9.5 & 486 & 437 & 49 & $\times$ \\
\hline Vim & 20 & 4.5 & 560 & 352 & 142 & 66 \\
& & 6 & 449 & 319 & 130 & $\times$ \\
& & 9.5 & 387 & 305 & 82 & $\times$ \\
& 40 & 4.5 & 516 & 287 & 29 & 200 \\
& & 6 & 188 & 188 & $\times$ & $\times$ \\
& & 9.5 & 478 & 187 & 163 & 128
\end{tabular}

Because PNIPAM is well-known from its shrinkage caused by temperature and $\mathrm{pH}$, the shrinkage parameter $\alpha$ is introduced and given by Eq. (4.1):

$$
\alpha=R_{\mathrm{h}} / R_{\mathrm{h}}^{0},
$$

where $R_{\mathrm{h}}$ is the hydrodynamic radius of PNIPAM copolymer obtained at $40^{\circ} \mathrm{C}$ and $R_{\mathrm{h}}^{0}$ - at $20^{\circ} \mathrm{C}$. The shrinkage parameter provides information about the magnitude of the radius decrease. The results showed in Fig. 4 that the heating of PNIPAM causes the shrinkage of particles. The magnitude of the shrinkage depends on the kind of copolymer. PNIPAM-ALA is insensitive to the $\mathrm{pH}$ and shrinks in the same ways in all tested $\mathrm{pH}$. For PNIPAM-Vim there is observed the slight increase of hydrodynamic radius in $\mathrm{pH}=9.5$.

The SLS tests confirmed that over LCST shell or shells disappeared and the core decreased for PNIPAM- 
-ALA and PNIPAM-MAA and PNIPAM-Vim ( $\mathrm{pH} 6)$. The results for PNIPAM-Vim ( $\mathrm{pH} 4$ and $\mathrm{pH}$ 9) showed the increase of shell layers that affected the increase of hydrodynamic radius, however the core size decreased. To confirm the assumption of parameter as a sphere scanning electron microscopy investigations were done. Figure 5 presents the structures of the copolymers. PNIPAM-MAA was uniformly distributed on the whole area, particles were not aggregated. PNIPAM-ALA and PNIPAM-Vim samples tend to aggregate, however the $\mathrm{pH}$ of samples was not controlled in these experiments. Aggregation process might be induced by high temperature of drying (over $50^{\circ} \mathrm{C}$ ).

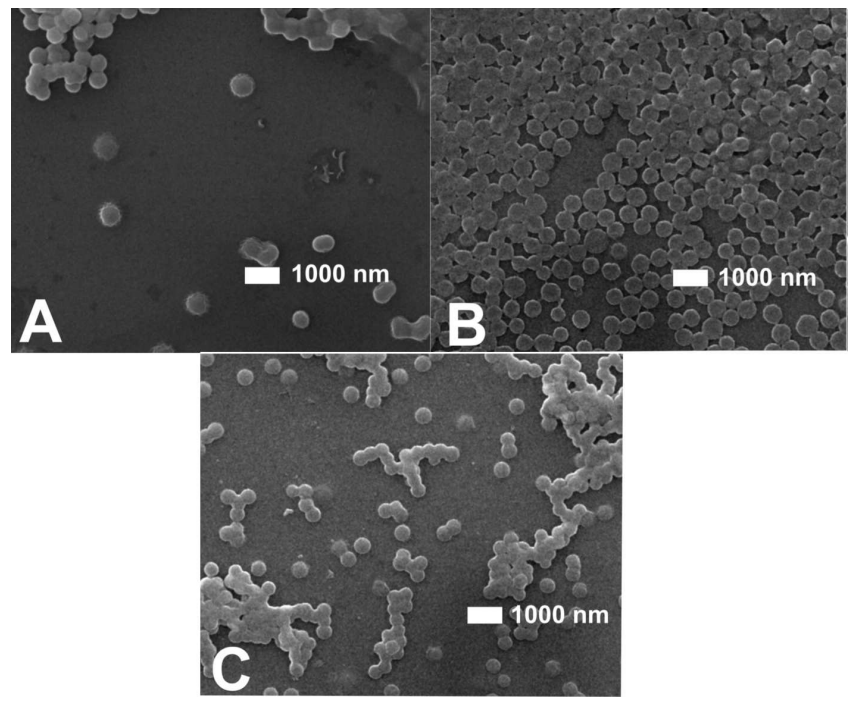

Fig. 5. SEM pictures of investigated copolymers: (A) PNIPAM-ALA, (B) PNIPAM-MAA, (C) PNIPAM-Vim (magnification $5000 \times$ ).

\section{Conclusions}

The most common change in structure of PNIPAM copolymers molecules is shrinkage induced by temperature. The performed tests showed quantitatively different reaction on temperature change depending on the $\mathrm{pH}$. PNIPAM-Vim selectively reacts on $\mathrm{pH}$. In $\mathrm{pH}=6$ the particle radius increased over 3 times. For PNIPAM-MAA the $\mathrm{pH}$ where the changes in radius were the highest was $\mathrm{pH}=4.5$.

SEM pictures showed that two PNIPAM copolymers: PNIPAM-ALA and PNIPAM-Vim tend to aggregate. However, the samples used in these experiments were not stabilized by buffer and dried in temperature over $50^{\circ} \mathrm{C}$. Further test performed on SEM with pH-controlled environment before drying should be performed. The results obtained in this part of research would be important for application of PNIPAM based copolymers in some systems induced by temperature in $\mathrm{pH}$.

\section{Acknowledgments}

Tomasz Śliwa thanks the NanoBioMedical Centre in Poznań, Adam Mickiewicz University, Faculty of Physics that the Ph.D. thesis is carried under the project co-financed by European Union, program Human Capital National Cohesion Strategy founded by European Social Fund.

Maciej Jarzębski thanks the Foundation for Polish Science and NanoBioMedical Centre in Poznań, Adam Mickiewicz University, Faculty of Physics, that the work was supported by the International Ph.D. Projects Programme of Foundation for Polish Science operated within the Innovative Economy Operational Programme (IE OP) 2007-2013 within European Regional Development Fund.

Financial support from the National Centre for Research and Development under research grant "Nanomaterials and Their Application to Biomedicine", contract number PBS1/A9/13/2012, is gratefully acknowledged.

\section{References}

[1] S. Chen, H. Zhong, B. Gu, Y. Wang, X. Li, Z. Cheng, L. Zhang, C. Yao, Mater. Sci. Eng. C-Mater. Biol. Appl. 32, 2199 (2012).

[2] C.Y. Chen, C.T. Chen, Chem. Commun. 47, 994 (2011).

[3] J. Meid, F. Dierkes, J. Cui, R. Messing, A.J. Crosby, A. Schmidt, W. Richtering, Soft Matter 8, 4254 (2012).

[4] A. Pich, W. Richtering, Adv. Polym. Sci. 234, 11 (2010).

[5] W. Richtering, Langmuir 28, 17218 (2012).

[6] H. Kawaguchi, K. Fujimoto, Y. Mizuhara, Coll. Polym. Sci. 270, 53 (1992).

[7] M. Yasui, T. Shiroya, K. Fujimoto, H. Kawaguchi, Coll. Surf. B, Biointerf. 8, 311 (1997).

[8] Y. Matsumaru, A. Hyodo, T. Nose, S. Ito, T. Hirano, S. Ohashi, J. Biomater. Sci. Polym. Ed. 7, 795 (1996).

[9] P.C. Naha, K. Bhattacharya, T. Tenuta, K.A. Dawson, I. Lynch, A. Gracia, F.M. Lyng, H.J. Byrne, Toxicol. Lett. 198, 134 (2010).

[10] S. Ito, K. Ogawa, H. Suzuki, B.L. Wang, R. Yoshida, E. Kokufuta, Langmuir 15, 4289 (1998).

[11] A.M. Santos, A. Elaissari, J.M.G. Martinho, C. Pichot, Polymer 46, 1181 (2005). 